OPEN ACCESS

Check for updates

\title{
Association between consumption of ultra-processed foods and all cause mortality: SUN prospective cohort study
}

\author{
Anaïs Rico-Campà, ${ }^{1,2}$ Miguel A Martínez-González, ${ }^{1,2,3,4}$ Ismael Alvarez-Alvarez, ${ }^{1}$ \\ Raquel de Deus Mendonça, ${ }^{1,5}$ Carmen de la Fuente-Arrillaga, ${ }^{1,2,3}$ Clara Gómez-Donoso, ${ }^{1}$ \\ Maira Bes-Rastrollo
}

${ }^{1}$ Department of Preventive Medicine and Public Health, University of Navarra, Irunlarrea 1 31008 Pamplona, Spain ${ }^{2}$ Biomedical Research Centre Network on Obesity and Nutrition (CIBERobn), Spanish National Institute of Health Carlos III, Madrid, Spain ${ }^{3}$ IDISNA, Navarra Institute for Health Research, Pamplona, Spain

${ }^{4}$ Harvard TH Chan School of Public Health, Boston, MA, USA ${ }^{5}$ Department of Nutrition, School of Nursing, Federal University of Minas Gerais, Belo Horizonte, Brazil

Correspondence to:

M Bes-Rastrollo mbes@unav.es (ORCID 0000-0002-9139-4206) Cite this as: BMJ 2019;365:1949 http://dx.doi.org/10.1136/bmj.l1949

Accepted: 23 April 2019

\section{ABSTRACT}

\section{OBJECTIVE}

To evaluate the association between consumption of ultra-processed foods and all cause mortality.

DESIGN

Prospective cohort study.

SETTING

Seguimiento Universidad de Navarra (SUN) cohort of university graduates, Spain 1999-2018.

\section{PARTICIPANTS}

19899 participants (12113 women and 7786 men) aged 20-91 years followed-up every two years between December 1999 and February 2014 for food and drink consumption, classified according to the degree of processing by the NOVA classification, and evaluated through a validated 136 item food frequency questionnaire.

\section{MAIN OUTCOME MEASURE}

Association between consumption of energy adjusted ultra-processed foods categorised into quarters (low, low-medium, medium-high, and high consumption) and all cause mortality, using multivariable Cox proportional hazard models.

RESULTS

335 deaths occurred during 200432 persons years of follow-up. Participants in the highest quarter (high consumption) of ultra-processed foods consumption had a higher hazard for all cause mortality compared with those in the lowest quarter (multivariable adjusted hazard ratio $1.62,95 \%$ confidence interval 1.13 to 2.33) with a significant dose-response relation ( $P$ for linear trend $=0.005$ ). For each additional serving of ultra-processed foods, all cause mortality relatively increased by $18 \%$ (adjusted hazard ratio $1.18,95 \%$ confidence interval 1.05 to 1.33 ).

CONCLUSIONS

A higher consumption of ultra-processed foods (>4 servings daily) was independently associated

\section{WHAT IS ALREADY KNOWN ON THIS TOPIC}

Ultra-processed foods are mostly or entirely made from substances derived from food and additives

Prospective cohort studies in adults found a link between the consumption of ultra-processed foods and a higher hazard of developing cancer, irritable bowel syndrome, obesity, and hypertension

\section{WHAT THIS STUDY ADDS}

A higher consumption of ultra-processed foods ( $>4$ servings daily) was independently associated with a $62 \%$ relatively increased hazard for all cause mortality Each additional serving of ultra-processed food daily increased the mortality hazard by $18 \%$ with a $62 \%$ relatively increased hazard for all cause mortality. For each additional serving of ultraprocessed food, all cause mortality increased by $18 \%$. STUDY REGISTRATION ClinicalTrials.gov NCT02669602.

\section{Introduction}

Nutrition is widely recognised as a crucial driver of chronic disease. ${ }^{1}$ Dietary habits influence many risk factors for cardiometabolic health, leading to type 2 diabetes, stroke, and heart disease, which are among the leading causes of death globally. Collectively, these risk factors associated with poor quality diet pose substantial health and economic burdens, and studies have shown that dietary factors are one of the main causes of the global burden of disease (measured as disability adjusted life years). ${ }^{2}$

The World Health Organization developed a Global Non-communicable Diseases (NCDs) Action Plan for 2013-2020, ${ }^{34}$ with the goal of achieving a $25 \%$ reduction in premature mortality from the four main global non-communicable diseases (cardiovascular disease, chronic respiratory disease, cancer, and diabetes) by 2025 . The global targets include improving the prevalence of risk factors (obesity, diabetes, raised blood pressure, tobacco use, salt intake, physical inactivity, and harmful use of alcohol). Global modelling of the impacts of risk factors on non-communicable diseases shows that premature mortality from the four main types between 2010 and 2025 could be reduced by $22 \%$ in men and $19 \%$ in women if the targets are achieved. ${ }^{5}$

During the past two decades, availability and consumption of ultra-processed foods, characterised by food products with a low nutritional quality and high energy density, has increased markedly in many countries. ${ }^{67}$ Between 1990 and 2010 the consumption of ultra-processed foods almost tripled (from 11\% to $32 \%$ of daily energy intake), ${ }^{8}$ parallel with increases in added sugar content. Ultra-processed foods are industrial formulations made mostly or entirely from substances derived from foods and additives, with little, if any, intact food. ${ }^{9}$ These food products are convenient (durable, ready to eat, or heatable), hyperpalatable (extremely tasty), highly profitable (low cost ingredients), and designed to replace all other food groups with the aid of attractive packaging and intensive marketing. ${ }^{10}$

Foods were first classified according to their degree of processing in 2010 using the NOVA system, which was last updated in $2016 .{ }^{9}$ Studies based on NOVA have 
shown an exponential growth in the consumption of ultra-processed foods. Negative nutritional attributes of ultra-processed food (high content of poor quality fat, added sugar and salt, along with low vitamin density and scarce fibre content) not only have a direct harmful effect on consumer's health but also affect health indirectly by replacing unprocessed or minimally processed foods and freshly prepared meals.

The beneficial effects of dietary patterns based on fresh or minimally processed foods on mortality are well known, but few studies have described the detrimental effects of high consumption of ultraprocessed foods. A meta-analysis found a statistically significant association between consumption of some specific ultra-processed foods (ie, sugar sweetened beverages, ${ }^{11}$ red meat, and processed meat ${ }^{12}{ }^{13}$ ) and mortality. In the French NutriNet-Santé cohort, authors found statistically significant associations between a higher consumption of ultra-processed foods and an increased risk of cancer ${ }^{14}$ and irritable bowel syndrome. ${ }^{15}$ In addition, early consumption of ultra-processed foods was associated with a higher incidence of dyslipidemia in Brazilian children ${ }^{16}$ and with a higher risk of overweight/obesity, ${ }^{17}$ and hypertension in a Spanish cohort. ${ }^{18}$

While we were responding to the reviewers' comments on our paper, the NutriNet-Santé cohort in France ${ }^{19}$ and the National Health and Nutrition Examination Survey (NHANES) cohort in the $\mathrm{US}^{20}$ also reported similar findings between consumption of ultra-processed foods and all cause mortality.

We assessed the relation between consumption of ultra-processed foods and mortality in the Seguimiento Universidad de Navarra (SUN) cohort.

\section{Methods}

\section{Study population}

The SUN project is a prospective, dynamic, and multipurpose cohort comprising Spanish university graduates. Its design, objectives, and methods have been described previously. ${ }^{21}$ Briefly, recruitment started in December 1999, and, as the project was designed to be a dynamic cohort, it is permanently open. Participants are followed-up every two years, with information gathered through postal or web-based questionnaires. To ensure a minimum follow-up of two years, we only considered participants recruited before March 2014 ( $n=22$ 279). We excluded 165 participants with a total daily energy intake below and above the first and 99th centiles, and 2215 participants were lost to follow-up (retention rate: 90\%). Data from 19899 participants were available for analyses.

\section{Dietary assessment}

Type of diet consumed was assessed at baseline with a 136 item semiquantitative food frequency questionnaire previously validated and repeatedly reevaluated in Spain. ${ }^{22-24}$ We measured frequencies of consumption in nine categories (ranging from never or almost never to more than six servings daily), and the food frequency questionnaire included a typical portion size for each item. To estimate daily consumption for each food item, we multiplied the portion size by the frequency of consumption.

We categorised all food and beverage items of the food frequency questionnaire into one of the four NOVA food groups-a classification system based on the extent and purpose of industrial food processing. ${ }^{9}$ The first group includes unprocessed or minimally processed foods, which are fresh or processed in ways that do not add substances such as salt, sugar, oils, or fats, and infrequently contain additives. The processes aim to extend life, allow storage for long use, and facilitate or enable different methods to be used for preparation (freezing, drying, and pasteurisation). Examples in this group include fruit and vegetables, grains (cereals), flours, nuts and seeds, fresh and pasteurised milk, natural yogurt with no added sugar or artificial sweeteners, meat and fish, tea, coffee, spices, and herbs. The second group contains processed culinary ingredients. These are substances obtained from foods of the first group or from nature and might contain additives to preserve the original properties (ie, salt, sugar, honey, vegetable oils, butter, lard, and vinegar). The third group comprises processed foods, to which substances such as salt, sugar, or oil have been added and methods such as smoking, curing, or fermentation have been used. Examples include canned or bottled vegetables and legumes, fruit in syrup, canned fish, cheeses, freshly made bread, and salted or sugared nuts and seeds. The fourth group comprises ultra-processed foods and drink products that are made predominantly or entirely from industrial substances and contain little or no whole foods. These products are ready to eat, drink, or heat-that is, carbonated drinks, sausages, biscuits (cookies), candy (confectionery), fruit yogurts, instant packaged soups and noodles, sweet or savoury packaged snacks, and sugared milk and fruit drinks. We focused on this last NOVA group.

To estimate the frequency of consumption of ultraprocessed food we summed the amount consumed (servings per day) of each food item classified in the fourth category of the NOVA system (a total of 34 items). We then divided the sample into quarters according to total consumption of ultra-processed foods (total servings per day). Box 1 shows the classification of the foods in the food frequency questionnaire according to NOVA. The food frequency questionnaire is a validated tool that can be used to assess total energy intake; macronutrient and fibre intake; alcohol intake; and consumption of fruit, vegetables, fast food, fried food, processed meat, unprocessed meat, and sugar sweetened beverages. ${ }^{22-24}$ Adherence to a Mediterranean diet was evaluated using the score proposed by Trichopoulou and colleagues. ${ }^{25}$

\section{Outcome assessment}

The primary outcome was all cause mortality. More than $85 \%$ of deaths were identified by reports from next of kin, work associates, and authority postal service. With permission of the next of kin, we reviewed the medical 
Box 1: Classification of foods in the SUN food frequency questionnaire according to degree of processing (NOVA)

Unprocessed or minimally processed foods
- Fruit, vegetables, legumes, milk (whole, semiskimmed, and non-fat), eggs, meats,
poultry, fish and seafood, fermented milk as yogurt, grains (white rice, pasta), natural
juice, coffee, and water
Processed culinary ingredients
- Salt, sugar, honey, vegetable oils (olive, sunflower, corn), chilli, butter, and lard
Processed foods
- Condensed milk, cream milk, cheeses, cured traditional ham, bacon, canned and
bottled fruit, breads (white and whole), beer, and wine
Ultra-processed foods
- Petit suisse; custard; flan; pudding; ice cream; ham; processed meat (chorizo, salami,
mortadella, sausage, hamburger, morcilla); pate; foie-gras; spicy sausage/meatballs;
potato chips; breakfast cereals; pizza, including pre-prepared pies; margarine;
cookies; chocolate cookies; muffins; doughnuts; croissant or other non-handmade
pastries; cakes; churros; chocolates and candies; nougat; marzipan; carbonated
drinks; artificially sugared beverages; fruit drinks; milkshakes; instant soups and
creams; croquettes; mayonnaise; and alcoholic drinks produced by fermentation
followed by distillation such as whisky, gin, and rum

records to confirm the deaths. To confirm the remainder of the deaths, we checked the Spanish National Death Index and the National Statistics Institute at least once a year. Given the continuous contact with participants in the cohort and the comprehensive and mandatory nature of the Spanish National Death Index, the use of these combined sources of information can be assumed to have $100 \%$ positive predictive value for fatal events.

Follow-up for each participant was calculated from the date when the baseline questionnaire was returned to the date of death or the date when the last follow-up questionnaire was returned, whichever came first. In only 22 out of 335 deaths $(6.5 \%)$ the cause of death was unknown.

\section{Assessment of other variables}

From the baseline questionnaire we also collected information on sex, age, marital status, educational level, smoking, physical activity, television viewing, napping, diet and dietary habits, and snacking. A validated 17 item questionnaire was used to evaluate physical activity. ${ }^{26}$ We also collected data on self reported anthropometric characteristics at baseline. A validation study with a subsample of the cohort showed sufficient validity for use in epidemiological studies. ${ }^{27}$ To detect underweight, overweight, and obesity we calculated the body mass index (BMI) as body weight $(\mathrm{kg})$ divided by height $\left(\mathrm{m}^{2}\right)$.

\section{Statistical analysis}

We used inverse probability weighting ${ }^{28}$ to adjust the means or proportions of baseline variables for age and sex according to quarters of consumption of ultra-processed foods. Consumption of ultra-processed food was adjusted for total energy intake using the residuals method and subsequently categorised into quarters: low consumption (first quarter), lowmedium consumption (second quarter), medium-high consumption (third quarter), and high consumption (fourth quarter). No data were missing for this variable of interest.

To assess the association between energy adjusted quarters of ultra-processed food consumption at baseline and all cause mortality, we fitted Cox regression models with age as the underlying time variable (birth date as origin), and date of death or date when the last follow-up questionnaire was completed for survivors as exit time. We estimated hazard ratios for the second to fourth quarters along with 95\% confidence intervals, with the lowest quarter as the reference category. To minimise the potential effect of a variation in diet during follow-up, we fitted Cox proportional hazard models with repeated dietary measurements using the updated data on food consumption after 10 years of follow-up.

We adjusted the Cox regression models for several potential confounders defined a priori. As recommended, we identified potential confounders based on existing literature, rather than deferring to statistical criteria. $^{29} 30$

Potential confounders included as covariates in multivariable models were age; sex; marital status, married (yes or no); baseline body mass index (linear and quadratic term); total energy intake (kcal/day, continuous); smoking status (never, current, former smoker); family history of cardiovascular disease (dichotomous); alcohol consumption (g/day, continuous); cardiovascular disease, cancer, or diabetes at baseline (yes or no); hypertension at baseline (yes or no); self reported hypercholesterolaemia at baseline (yes or no); depression at baseline (yes or no); educational level (non-graduate, graduate, postgraduate, doctorate); snacking (yes or no); following a special diet at baseline (yes or no); physical activity (quarters); and lifelong cumulative exposure to smoking (pack years of smoking, continuous). Results were stratified by recruitment period (1999-2000, 2001, 2002-03, 2004, 2005-07, 2008-14), deciles of age, time spent watching television (dichotomous, cut-off: $\geq 3 \mathrm{~h} /$ day), and four categories of a sedentary index defined as the number of hours spent daily watching television, using a computer, and driving. When participants had missing values on snacking or following a special diet, we considered them as doing neither, and we also used multiple imputation for missing values in those variables.

In addition to standard adjustment for confounders, we alternatively adjusted the models using propensity scores.

Although we adjusted for a wide range of confounders, we cannot rule out residual confounding. Consumption of ultra-processed food is a behaviour that might be closely linked to other aspects of a non-healthy lifestyle. To assess this in detail, we calculated the $\mathrm{E}$ value proposed by Vanderweele. ${ }^{31} 32$ This value represents the minimum strength of association, on the risk ratio scale, that an unmeasured confounder would need with both the exposure and the outcome, conditioned by the measured covariates, to fully explain a specific association. 
To investigate linear trends across the quarters of consumption of ultra-processed foods we assigned the median value to each category and considered the variable as being continuous. We verified the proportionality of hazards with a test based on Schoenfeld residuals; the non-significant result $(\mathrm{P}=0.11)$ suggested that the proportionality assumption had been met.

To assess the contribution of each food group to the total consumption of ultra-processed foods, we calculated the ratio between the servings of each food group divided by the total servings of ultra-processed foods multiplied by 100 .

We used Kaplan-Meier curves, with inverse probability weighting to adjust for confounding, to describe all cause mortality according to baseline quarters of ultra-processed foods consumption. To simplify the graph, we merged the first and second quarters (low and low-medium consumption) into one group and the third and fourth quarters (highmedium and high consumption) in another group. This grouping lowers random variability and provides more stable estimates.

Based on our experience and on several simulations, we used restricted cubic splines to calculate the potential non-parametrical non-linear association between consumption of ultra-processed food and all cause mortality. Tests for non-linearity used the likelihood ratio test to compare the model that comprised the linear term with the model that comprised both the linear and the cubic spline terms. The likelihood of both models can be compared using the Akaike's information criterion or bayesian information criterion. Both penalise the likelihood of the model, and the one that results in the lowest value using either criterion will be the most likely model.

Additionally, we conducted subgroup analyses by rerunning all the models under different a priori assumptions: including only men, only women, only participants aged 50 or older at recruitment, and only participants aged 50 or younger at recruitment; truncating the follow-up at three years; starting followup at three years after the baseline questionnaire; excluding participants with a BMI of less than 25 or 25 or more; including only never smokers; and excluding never smokers.

Sensitivity analyses were also conducted by rerunning the models under different a priori assumptions: using the 5th and 95th centiles as limits for allowable total energy intake; using energy limits previously proposed by Willett $^{33}$; excluding participants with prevalent cardiovascular disease or cancer; excluding participants with hypertension at baseline; excluding participants with depression at baseline; excluding participants following special diets at baseline; and excluding deaths from injuries, deaths from cancer, and deaths from cardiovascular disease. We additionally adjusted for weight gain of 3 $\mathrm{kg}$ or more in the year before inclusion in the cohort, coffee consumption, a quadratic term of alcohol intake, consumption of all fried foods, following a
Mediterranean diet, ${ }^{25}$ sodium intake, and intake of saturated and trans fatty acids, added sugars, and sodium.

We considered $\mathrm{P}$ values of less than 0.05 to be statistically significant, and these were corrected using Simes method. ${ }^{34}$ Analyses were performed using STATA version 15.0 (StataCorp, College Station, TX).

\section{Patient and public involvement}

No patients were involved in setting the research question or the outcome measures. The research question of this study was included in the context of the SUN cohort objectives. Participants involved in the SUN cohort are volunteers. The results of this study will be disseminated to the participants and the public through the cohort website, public sessions, and a press release.

\section{Results}

A total of 7786 men and 12113 women were included in this analysis (fig 1). Mean age at baseline was 37.6 (SD 12.3) years and duration of follow-up was a median of 10.4 years. Overall, 335 deaths occurred during 200432 person years of follow-up. Table 1 shows the baseline characteristics of participants according to quarters of total ultra-processed food consumption adjusted for sex and age.

Participants in the fourth quarter (high consumption of ultra-processed foods) had a higher average BMI. Compared with participants in the first quarter, they were more likely to be current smokers, to have a higher level of university education, and have a family history of cardiovascular disease, cancer, diabetes, hypertension, hypercholesterolaemia, prevalent cardiovascular disease, and depression. In addition, they were more likely to snack, watch television, and use a computer for longer periods, more prone to be sedentary and nap, and had higher total fat intake but lower protein and carbohydrate intake. On average they consumed more fast food, fried foods, processed meats, and sugar sweetened beverages than participants in the other quarters and had the lowest intake of vegetables, fruit, olive oil, alcohol, and total fibre. Adherence to the Mediterranean diet tended to be progressively lower across successive quarters of ultra-processed foods consumption (ie, the higher the consumption of ultra-processed foods, the lower the adherence to the Mediterranean diet), with a correlation coefficient $\mathrm{r}=-0.39$ (95\% confidence interval -0.40 to -0.38 ) between the score for adherence to the Mediterranean diet and consumption of ultra-processed foods. Processed meats, sugar sweetened beverages, dairy products, and French fries were the main foods contributing to the total of ultraprocessed food consumed (table 2).

The main cause of death was cancer $(n=164)$, with a mean age at death of 58.0 (SD 15.6) years. Participants in the highest quarter of ultra-processed food consumption had a $62 \%$ relatively higher hazard of all cause mortality compared with those in the lowest quarter (multivariable adjusted hazard ratio 1.62, 95\% 


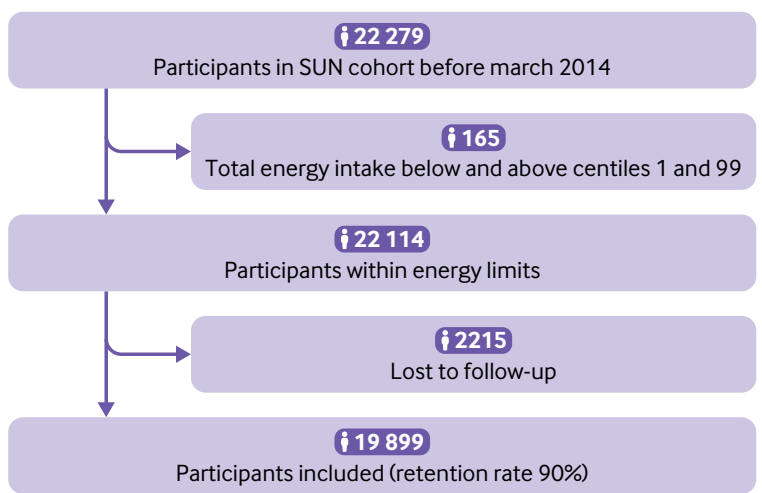

Fig 1 | Flowchart of study participants. SUN=Seguimiento Universidad de Navarra

confidence interval 1.13 to 2.33), with a significant dose-response relation ( $\mathrm{P}$ for trend $=0.005$ ) (table 3; fig 2). For each additional serving of ultra-processed food, all cause mortality relatively increased by $18 \%$ (adjusted hazard ratio 1.18, 95\% confidence interval 1.05 to 1.33 ).

When multiple imputation was used for those covariates with missing values the results were unchanged. A new Cox proportional hazard models was fitted with repeated measurements using the updated data on food consumption after 10 years of follow-up: the hazard ratio for the fourth versus the first quarter was 1.44 (95\% confidence interval 1.01 to 2.05), with a significant dose-response relation ( $\mathrm{P}$ for trend $=0.023$ ) (table 3 ). When propensity scores were alternatively used to adjust for potential residual confounding, the magnitude of the association between consumption of ultra-processed foods and mortality increased to 1.89 (1.34 to 2.67).

The observed hazard ratio of 1.62 in our main analysis could hypothetically be explained by the presence of an unmeasured confounder that was associated with consumption of ultra-processed food and all cause mortality by a hazard ratio of 2.62 -fold each, above and beyond the measured confounders, but weaker confounding could not do so. Similarly, the lowest confidence interval could be moved to include the null by an unmeasured confounder that was associated with both consumption of ultra-processed foods and all cause mortality by a hazard ratio of 1.51-fold each, above and beyond the measured confounders, but weaker confounding could not do so. ${ }^{3132}$

Subgroup analyses were carried out by repeating the multivariable adjusted Cox regression models in different scenarios comparing the highest with the lowest quarter of ultra-processed foods. Using the likelihood ratio test, the $\mathrm{P}$ for interaction was calculated between consumption of ultra-processed foods (quarters) and stratification variables for each scenario. None were significant $(\mathrm{P}=0.94$ for sex, $\mathrm{P}=0.37$ for age, $\mathrm{P}=0.98$ for years of follow-up, $\mathrm{P}=0.93$ for $\mathrm{BMI}$, and $\mathrm{P}=0.72$ for smoking status).

Sensitivity analyses were conducted by repeating the multivariable adjusted Cox regression models in different scenarios comparing the fourth quarter with the first quarter of ultra-processed foods. All point estimates showed a direct association between consumption of ultra-processed foods and higher mortality. Results did not substantially change in any of these alternative scenarios, suggesting that the direct association between consumption of ultraprocessed food and mortality was robust (fig 3). Some associations did, however, become non-significant under the scenarios of excluding cases of prevalent hypertension at baseline and excluding cases of depression at baseline. Conversely, the association became stronger after changing the energy limits to the 5th and 95th centiles and after excluding prevalent cardiovascular disease or cancer, deaths from injuries, and deaths from cancer.

When the harmful effect of consumption of ultraprocessed food on all cause mortality was assessed by ultra-processed food specific nutritional components, no evidence was found of a mediation effect. That is, the association was not reduced after additionally adjusting for saturated and trans fatty acids, added sugars, and sodium intake. Finally, the restricted cubic spline analysis-with three knots, and adjusted for the same potential confounders-suggested that the consumption of five or more servings of ultra-processed foods daily (which corresponds to the mean of the highest quarter) was associated with a significantly higher hazard of all cause mortality (fig 4).

\section{Discussion}

In this longitudinal cohort study of Spanish university graduates aged 20-91, consumption of ultra-processed foods was associated with an increased hazard for all cause mortality. While we were addressing the reviewers' comments on our paper, the NutriNetSanté $^{19}$ and the US National Health and Nutrition Examination Survey (NHANES) ${ }^{20}$ cohorts also reported similar associations. Similar results in different populations, with different age ranges (NutriNet-Santé cohort: $\geq 45$ years) and diverse methods for assessing dietary exposures support a causal association. ${ }^{19} 20$

In our study we found that a high consumption of ultra-processed foods ( $>4$ servings daily) was significantly associated with a $62 \%$ relatively higher hazard of mortality. Moreover, each additional serving of ultra-processed food was associated with a statistically significant $18 \%$ higher hazard of all cause mortality.

\section{Comparison with other studies}

TheNOVAclassification is clear, useful, understandable, and simple to apply. ${ }^{9}$ The methodology of the classification has been criticised, ${ }^{35}$ but we have not found a better alternative. It is also easily incorporated into messages and is reproducible and therefore has utility for public health. In addition, it is the best known and most frequently used classification of ultraprocessed food in epidemiological studies.

We used the NOVA classification to identify four different food groups according to the degree of 


\begin{tabular}{|c|c|c|c|c|}
\hline \multirow[b]{2}{*}{ Characteristics } & \multicolumn{4}{|c|}{ Quarters of energy adjusted ultra-processed food consumption } \\
\hline & $\begin{array}{l}\text { First }(<2 \text { servings/day }) \\
(\mathrm{n}=4975)\end{array}$ & $\begin{array}{l}\text { Second ( } 2-<3 \text { servings/day) } \\
(n=4975)\end{array}$ & $\begin{array}{l}\text { Third (3- } \leq 4 \text { servings/day) } \\
(n=4975)\end{array}$ & $\begin{array}{l}\text { Fourth (>4 servings/day) } \\
(\mathrm{n}=4974)\end{array}$ \\
\hline Ultra-processed foods (servings/day) & $1.4(0.8)$ & $2.7(0.2)$ & $3.5(0.3)$ & $5.3(1.4)$ \\
\hline Body mass index & $23.3(3.4)$ & $23.5(3.5)$ & $23.6(3.7)$ & $23.8(3.7)$ \\
\hline Married & $2520(50.8)$ & $2487(50.4)$ & $2481(50.0)$ & $2505(49.6)$ \\
\hline \multicolumn{5}{|l|}{ Educational level (No (\%)): } \\
\hline Graduate & $4192(84.6)$ & $4047(82.0)$ & 4019 (81.1) & $4084(80.9)$ \\
\hline Postgraduate & $328(6.6)$ & $385(8.0)$ & $412(8.3)$ & $417(8.3)$ \\
\hline Doctorate & $435(8.8)$ & $506(10.3)$ & $525(10.6)$ & $549(10.9)$ \\
\hline \multicolumn{5}{|l|}{ Smoking status (No (\%)): } \\
\hline Current & $1141(23.0)$ & $1194(24.2)$ & $1231(24.8)$ & $1426(28.3)$ \\
\hline Former & $1313(26.5)$ & $1265(25.6)$ & $1203(24.3)$ & $1215(24.1)$ \\
\hline Family history of CVD (No (\%)) & $667(13.5)$ & $684(13.9)$ & $679(13.7)$ & $802(15.9)$ \\
\hline \multicolumn{5}{|l|}{ Conditions at baseline (No (\%)): } \\
\hline Cancer & $173(3.5)$ & $159(3.2)$ & $170(3.4)$ & $224(4.4)$ \\
\hline Diabetes & $98(2.0)$ & $91(1.8)$ & $100(2.1)$ & $116(2.3)$ \\
\hline Hypertension & $935(18.8)$ & $980(19.9)$ & $975(19.7)$ & $1148(22.7)$ \\
\hline Hypercholesterolaemia & $856(17.3)$ & $840(17.0)$ & $854(17.2)$ & $898(17.8)$ \\
\hline Cardiovascular disease & $76(1.5)$ & $73(1.5)$ & $78(1.6)$ & $122(2.4)$ \\
\hline Depression & $535(10.8)$ & $553(11.2)$ & $594(12.0)$ & $677(13.4)$ \\
\hline Following a special diet (No (\%)) & $423(8.5)$ & $415(8.4)$ & $407(8.2)$ & $366(7.2)$ \\
\hline Snacking (No (\%)) & $1461(29.5)$ & $1527(30.9)$ & $1677(33.8)$ & $2139(42.4)$ \\
\hline \multicolumn{5}{|l|}{ Sedentary activities: } \\
\hline Television viewing ( $\geq 3 \mathrm{~h} /$ day) & $340(6.9)$ & $359(7.3)$ & $407(8.2)$ & $530(10.5)$ \\
\hline Computer use (h/day) & $2.0(1.9)$ & $2.1(1.9)$ & $2.1(1.9)$ & $2.2(2.0)$ \\
\hline Driving (h/day) & $0.9(1.1)$ & $0.9(1.1)$ & $0.9(1.1)$ & $0.9(1.1)$ \\
\hline Napping (h/day) & $0.3(0.7)$ & $0.3(0.8)$ & $0.3(0.8)$ & $0.4(0.8)$ \\
\hline Sedentary indext (h/day) & $4.5(2.8)$ & $4.6(2.8)$ & $4.7(2.6)$ & $4.9(2.8)$ \\
\hline Physical activity (MET hours weekly) & $30.8(27.6)$ & $27.1(23.0)$ & $25.5(22.0)$ & $25.2(23.8)$ \\
\hline Adherence to Mediterranean diet (0-9 score) & $5.1(1.7)$ & $4.3(1.7)$ & $3.8(1.7)$ & $3.6(1.7)$ \\
\hline Total energy intake (kcal/day) & $2799(764.3)$ & $2338(693.1)$ & $2299(714.7)$ & $2632(873.0)$ \\
\hline \multicolumn{5}{|l|}{ Macronutrients intake (\% energy): } \\
\hline Carbohydrate & $44.6(7.9)$ & $42.9(7.3)$ & $42.8(7.0)$ & $43.6(7.5)$ \\
\hline Protein & $18.1(3.4)$ & $18.6(3.3)$ & $18.3(3.1)$ & $16.9(3.1)$ \\
\hline Fat: & $35.3(7.2)$ & $36.4(6.4)$ & $37.0(6.0)$ & $37.5(6.5)$ \\
\hline SFAs & $11.5(3.4)$ & $12.3(3.0)$ & $12.8(3.0)$ & $13.2(3.2)$ \\
\hline MUFAs & $15.6(4.1)$ & $15.8(3.7)$ & $15.7(3.4)$ & $15.6(3.5)$ \\
\hline PUFAs & $5.0(1.6)$ & $5.1(1.5)$ & $5.3(1.5)$ & $5.4(1.7)$ \\
\hline Total dietary fibre intake (g/day) & $37.9(17.1)$ & $28.6(11.5)$ & $26.0(11.0)$ & $26.5(12.7)$ \\
\hline Alcohol consumption (g/day) & $7.5(12.0)$ & $6.6(9.5)$ & $6.0(9.5)$ & $7.3(12.3)$ \\
\hline Olive oil (g/day) & $22.5(17.5)$ & $15.9(13.2)$ & $13.2(11.9)$ & $12.7(12.1)$ \\
\hline \multicolumn{5}{|l|}{ Food consumption (servings/day): } \\
\hline Fruit & $4.3(3.2)$ & $2.9(2.0)$ & $2.5(1.8)$ & $2.4(1.9)$ \\
\hline Vegetables & $3.5(2.2)$ & $2.8(1.4)$ & $2.5(1.3)$ & $2.4(1.5)$ \\
\hline Fast food $\neq$ & $0.1(0.1)$ & $0.2(0.2)$ & $0.2(0.2)$ & $0.2(0.2)$ \\
\hline Fried food & $0.5(0.7)$ & $0.5(0.6)$ & $0.5(0.6)$ & $0.6(0.7)$ \\
\hline Red meat & $0.6(0.4)$ & $0.6(0.3)$ & $0.5(0.3)$ & $0.5(0.3)$ \\
\hline Processed meat§ & $0.5(0.5)$ & $0.6(0.4)$ & $0.8(0.5)$ & $1.0(0.8)$ \\
\hline Sugar sweetened beverages & $0.1(0.2)$ & $0.2(0.3)$ & $0.3(0.4)$ & $0.8(1.2)$ \\
\hline Low fat dairy products & $0.5(1.5)$ & $0.4(0.8)$ & $0.4(0.8)$ & $0.4(0.9)$ \\
\hline High fat dairy products & $0.6(1.1)$ & $0.5(0.8)$ & $0.4(0.7)$ & $0.4(0.7)$ \\
\hline \multicolumn{5}{|l|}{ Mineral intake (mg/day): } \\
\hline Sodium & $4103(2032)$ & $3783(2116)$ & $4053(2578)$ & $4909(4248)$ \\
\hline Potassium & $6122(2187)$ & $4887(1544)$ & $4560(1516)$ & $4630(1721)$ \\
\hline Calcium & $1531(661)$ & $1242(480)$ & $1176(473)$ & $1246(518)$ \\
\hline Magnesium & $527(166)$ & $421(125)$ & $398(124)$ & $421(149)$ \\
\hline Phosphorous intake (mg/day) & $2346(729)$ & $1953(560)$ & $1869(563)$ & $1970(641)$ \\
\hline Caffeine intake (mg/day) & $40.9(39.2)$ & $40.2(37.0)$ & $40.0(36.2)$ & $52.0(49.0)$ \\
\hline
\end{tabular}

MET=metabolic equivalent of task; SFAs=saturated fatty acids; MUFAs=monounsaturated fatty acids; PUFAs=polyunsaturated fatty acids.

${ }^{*}$ Adjusted through inverse probability weighting.

tSum of hours each day spent watching television, using a computer, and driving.

‡Sum of hamburgers, sausages, and pizza.

$\S$ Sum of ham, sausages, chorizo, salami, mortadella, and hamburgers.

processing. Our interest was in the fourth group of the NOVA system, which included ultra-processed food and drink products that tend to be nutritionally unbalanced as a result of undergoing several industrial processes. These foods are economically profitable because the shelf life, and therefore sales, of these 


\begin{tabular}{|c|c|}
\hline Food groups & Contribution (\%) \\
\hline Processed meats* & 15 \\
\hline Sugar sweetened beverages & 15 \\
\hline Dairy products $t$ & 12 \\
\hline French fries & 11 \\
\hline Pastries $\neq$ & 10 \\
\hline Cookies§ & 8 \\
\hline Ready to eat soups and purées & 6 \\
\hline Fried foods & 6 \\
\hline Artificially sugared beverages & 5 \\
\hline Breakfast cereals & 3 \\
\hline Pizza & 2 \\
\hline Liquors & 2 \\
\hline Margarine & 1 \\
\hline Mayonnaise & 1 \\
\hline \multicolumn{2}{|c|}{$\begin{array}{l}\text { SUN=Seguimiento Universidad de Navarra. } \\
\text { *Includes ham, sausages, chorizo, salami, mortadella, and hamburgers } \\
\text { tIncludes custard, ice cream, milkshakes, and petit suisse. } \\
\text { fIncludes muffins, doughnuts, croissants or other non-handmade } \\
\text { pastries, and confectionery. } \\
\text { SIncludes biscuits and chocolate cookies. }\end{array}$} \\
\hline
\end{tabular}

food products are increased, but nutritional quality is decreased, characterised by a high energy density, low fibre and micronutrient content, and high amounts of added or free sugars, sodium, saturated fat, and chemical additives. ${ }^{10}$

In the past two decades, the intake of ready-toeat, ready-to-drink, and ready-to-heat "fast" and "convenient" products has noticeably increased in all countries, regardless of economical level. This might have contributed to the global increased rates of overall cancer, ${ }^{14}$ dyslipidemia, ${ }^{16}$ obesity, ${ }^{17}$ and hypertension. ${ }^{18}$

Two recent prospective French studies using data from the NutriNet-Santé cohort evaluated the relation between consumption of ultra-processed foods and risk of overall cancer and gastrointestinal disorders. These studies found a positive association between increased consumption of ultra-processed food and risk of cancer overall, breast cancer, ${ }^{14}$ and irritable bowel syndrome. ${ }^{15}$ Moreover, previous results in the SUN cohort found that consumption of ultra-processed food was associated with a higher risk of overweight, obesity, and hypertension. ${ }^{17} 18$ These findings seem to be in line with our present study and consistently show the adverse effects related to consumption of ultraprocessed food.

Other findings from a large national cross sectional study, the United Kingdom National Diet and Nutrition

\begin{tabular}{|c|c|c|c|c|c|}
\hline \multirow[b]{2}{*}{ Variables } & \multicolumn{4}{|c|}{ Quarters of energy adjusted ultra-processed foods consumption } & \multirow[b]{2}{*}{ P for trend } \\
\hline & First (<2 servings/day) & Second $(2-<3$ servings/day) & Third ( $3-\leq 4$ servings/day) & Fourth ( $>4$ servings/day) & \\
\hline \multicolumn{6}{|l|}{ All cause mortality } \\
\hline No of participants & 4975 & 4975 & 4975 & 4974 & \\
\hline Person years & 49814 & 50322 & 49971 & 50323 & \\
\hline No of deaths & 108 & 74 & 80 & 73 & \\
\hline Unadjusted & 1.00 (reference) & $1.02(0.75$ to 1.37$)$ & $1.38(1.03$ to 1.85$)$ & $1.78(1.30$ to 2.43$)$ & $<0.001$ \\
\hline Age and sex adjusted & 1.00 (reference) & $1.00(0.73$ to 1.36$)$ & $1.29(0.94$ to 1.77$)$ & $1.72(1.22$ to 2.43$)$ & 0.001 \\
\hline Multivariable adjusted ${ }^{\star}$ & 1.00 (reference) & $0.99(0.72$ to 1.37$)$ & $1.24(0.89$ to 1.73$)$ & $1.61(1.12$ to 2.30$)$ & 0.008 \\
\hline Multivariable adjusted $t$ & 1.00 (reference) & $1.06(0.76$ to 1.48$)$ & $1.38(0.99$ to 1.92$)$ & $1.62(1.13$ to 2.33$)$ & 0.005 \\
\hline Adjusted for propensity scores§ & 1.00 (reference) & $1.07(0.78$ to 1.46$)$ & $1.43(1.01$ to 1.98$)$ & 1.89 (1.34 to 2.67$)$ & $<0.001$ \\
\hline Repeated measurements of diet & 1.00 (reference) & $1.18(0.85$ to 1.63$)$ & 1.39 (1.00 to 1.93$)$ & 1.44 (1.01 to 2.05$)$ & 0.02 \\
\hline \multicolumn{6}{|l|}{ Cardiovascular deaths } \\
\hline No of participants & 4889 & 4921 & 4909 & 4916 & \\
\hline Person years & 49245 & 49941 & 49474 & 49893 & \\
\hline No of deaths & 22 & 20 & 14 & 15 & \\
\hline Unadjusted & 1.00 (reference) & 1.37 (0.74 to 2.53$)$ & $1.43(0.73$ to 2.76$)$ & $1.86(0.90$ to 3.83$)$ & 0.09 \\
\hline Age and sex adjusted & 1.00 (reference) & 1.04 (0.54 to 2.00$)$ & $1.33(0.62$ to 2.87$)$ & 2.08 (0.96 to 4.52$)$ & 0.17 \\
\hline Multivariable adjusted* & 1.00 (reference) & $0.87(0.41$ to 1.84$)$ & $1.13(0.45$ to 2.82$)$ & $2.10(0.94$ to 4.69$)$ & 0.10 \\
\hline Multivariable adjustedt & 1.00 (reference) & $0.77(0.32$ to 1.83$)$ & $1.14(0.46$ to 2.82$)$ & $2.16(0.92$ to 5.06$)$ & 0.11 \\
\hline Repeated measurements of diet & 1.00 (reference) & 0.95 (0.41 to 2.22) & $1.40(0.56$ to 3.50$)$ & $1.94(0.82$ to 4.61$)$ & 0.14 \\
\hline \multicolumn{6}{|l|}{ Cancer deaths } \\
\hline No of participants & 4929 & 4934 & 4935 & 4930 & \\
\hline Person years & 49518 & 50019 & 49689 & 49987 & \\
\hline No of deaths & 62 & 33 & 40 & 29 & \\
\hline Unadjusted & 1.00 (reference) & $0.78(0.51$ to 1.20$)$ & $1.26(0.85$ to 1.86$)$ & $1.36(0.85$ to 2.18$)$ & 0.14 \\
\hline Age and sex adjusted & 1.00 (reference) & $0.80(0.52$ to 1.23$)$ & $1.03(0.68$ to 1.56$)$ & $1.36(0.82$ to 2.27$)$ & 0.20 \\
\hline Multivariable adjusted ${ }^{\star}$ & 1.00 (reference) & 0.79 (0.51 to 1.23$)$ & $1.01(0.65$ to 1.57$)$ & $1.23(0.71$ to 2.11$)$ & 0.50 \\
\hline Multivariable adjusted $\dagger$ & 1.00 (reference) & $0.87(0.55$ to 1.38$)$ & $1.13(0.73$ to 1.73$)$ & $1.22(0.70$ to 2.12$)$ & 0.42 \\
\hline Repeated measurements of diet & 1.00 (reference) & 0.97 (0.60 to 1.57) & $1.43(0.93$ to 2.20$)$ & $1.30(0.77$ to 2.21$)$ & 0.17 \\
\hline \multicolumn{6}{|c|}{$\begin{array}{l}\text { *Adjusted for age (underlying time variable), sex, marital status, physical activity (quarters), smoking status (never, current, former), snacking (dichotomous), special diet at baseline } \\
\text { (dichotomous), body mass index (linear and quadratic terms), total energy intake (continuous), alcohol consumption (continuous), and educational level (continuous) stratified by recruitment } \\
\text { period, deciles of age, sedentary index (sum of hours each day spent watching television, using a computer, and driving), and television viewing ( } \geq 3 \mathrm{~h} / \text { day). } \\
\text { tAdjusted for age (underlying time variable), sex, marital status, physical activity (quarters), smoking status (never, current, former), snacking (dichotomous), special diet at baseline } \\
\text { (dichotomous), body mass index (linear and quadratic terms), total energy intake (continuous), alcohol consumption (continuous), family history of cardiovascular disease (CVD, dichotomous), } \\
\text { diabetes at baseline (dichotomous), hypertension at baseline (dichotomous), self reported hypercholesterolaemia at baseline (dichotomous), CVD at baseline (dichotomous), cancer at baseline } \\
\text { (dichotomous), depression at baseline (dichotomous), education level (continuous) and lifelong smoking (pack-years of smoking, continuous) stratified by recruitment period, deciles of age, } \\
\text { sedentary index (sum of hours each day spent watching television, using a computer, and driving), and television viewing ( } \geq 3 \mathrm{~h} / \text { day). } \\
\text { tSum of hours each day spent watching television, using a computer, and driving. } \\
\text { SMultivariable adjusted for propensity scores. } \\
\text { qMultivariable adjusted model with repeated measures (updated data at } 10 \text { years of follow-up). }\end{array}$} \\
\hline
\end{tabular}



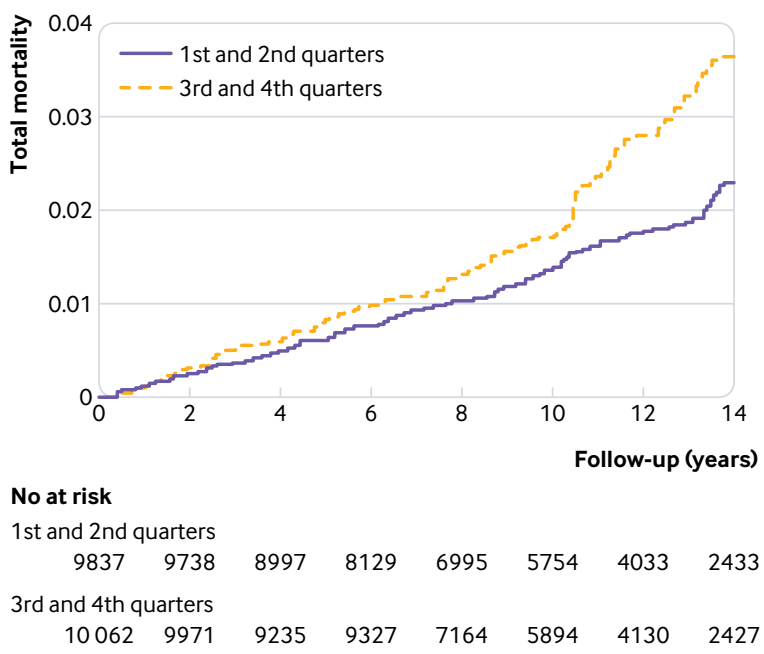

Fig 2 | Kaplan-Meier incidence for consumption of ultra-processed foods, adjusted through inverse probability weighting. See table 3 footnote $t$ for adjustment factors

Survey, showed that diets rich in unprocessed foods and low in ultra-processed foods are associated with a healthier food profile, although no association was found for body weight. ${ }^{36}$ Several studies have reported other adverse effects related to consumption of ultra-processed food. In the framework of NHANES, authors reported a strong inverse association between consumption of ultra-processed food and urinary concentrations of phytoestrogens. ${ }^{37}$ A longitudinal study of pregnant women in the United States suggested that the percentage of total calorie intake from ultra-processed food might be a useful predictor of gestational weight gain and neonatal body fat. ${ }^{38}$

In another US study the availability of ultraprocessed foods in households was analysed and showed that $61 \%$ of total purchased dietary energy was derived from such foods. ${ }^{39}$ A cross sectional study using data from NHANES found that ultra-processed foods represented $58 \%$ of energy intake and that $90 \%$ of this energy was derived from added sugars. ${ }^{40}$ Similarly, results from the 2004 Pelotas (Brazil) Birth Cohort Study showed that $40 \%$ of total daily energy intake in six year old children was from ultra-processed foods. $^{41}$

Altogether these results support the non-beneficial effects of ultra-processed foods, which currently represent a major portion of the calories consumed in many countries. In line with the cited evidence, our findings reinforce the existing evidence on the negative impact of ultra-processed foods on the overall incidence of chronic diseases and all cause mortality.

\section{Strengths and limitations of this study}

The strengths of this study are its prospective and dynamic design, the use of validated methods, adjustment for a wide range of potential confounders, long follow-up, good retention rate (90\% overall), and use of a variety of sensitivity analyses to support the robustness of the results. Although the findings were based on self reports, we can assume that the data are of high quality because the participants were highly motivated university graduates, which adds validity to the information derived from their questionnaires and reduces the potential misclassification bias. Another strength of our longitudinal study is its novelty. The consistency of the relation between consumption of ultra-processed food and all cause mortality that we detected was simultaneously reported by the NutriNetSanté study and the NHANES cohort. ${ }^{1920}$

Our study, however, has limitations. The food frequency questionnaire was not specifically designed to collect data about the new NOVA classification of ultra-processed food consumption. We did not include cereal and energy bars, energy drinks, health and slimming products, and meat or vegetable nuggets, because we did not have information on the consumption of these items between the food frequency questionnaire and the food records used in the validation study.

Moreover, using servings of ultra-processed food as an indicator for consumption could lead to misclassification. Nevertheless, in the validation of the SUN cohort the intraclass correlation coefficient ${ }^{24}$ for different ultra-processed foods were: processed meat $(\mathrm{r}=0.74)$, soft drinks $(\mathrm{r}=0.62)$, confectionery pastries $(\mathrm{r}=0.66)$, cookies $(\mathrm{r}=0.36)$, breakfast cereals $(\mathrm{r}=0.71)$, chocolate $(r=0.65)$, fast food $(r=0.42)$, fruit drinks in bottles $(r=0.88)$, margarine $(r=0.59)$, ice cream $(\mathrm{r}=0.65)$, and distilled liquors $(\mathrm{r}=0.71)$. These values are in the range of the estimates usually observed in studies of nutritional epidemiology.

As our study was observational, we cannot rule out residual confounding. We did, however, adjust for a wide range of potential confounders using different statistical methods, and the results were consistent. In addition, the $\mathrm{E}$ values for the point estimate supported the observed association. The point estimate could be theoretically explained only by an unmeasured confounder with a hazard ratio of at least 2.62-fold for mortality and for ultra-processed food consumption.

Another weakness is the limited external validity of our findings, as the cohort was not representative of the general population. Prevalence and incidence might not reflect those in Spain. Nevertheless, in analytical epidemiology, cohorts are usually non-representative, and therefore generalisation of these results must be based on biological mechanisms rather than on statistical representativeness. Finally, the number of observed deaths was small, and we acknowledge that some analyses can be underpowered, especially in analyses considering only cause specific mortality.

The SUN cohort is a relatively young population and it is restricted to university graduates with high educational level. Consequently, the sample is not representative of the general population. However, lack of representativeness does not prevent associations that can be generalised to other groups from being established, provided no biological mechanism suggests that the association no longer holds for other populations. ${ }^{42-44}$ We did not control for 


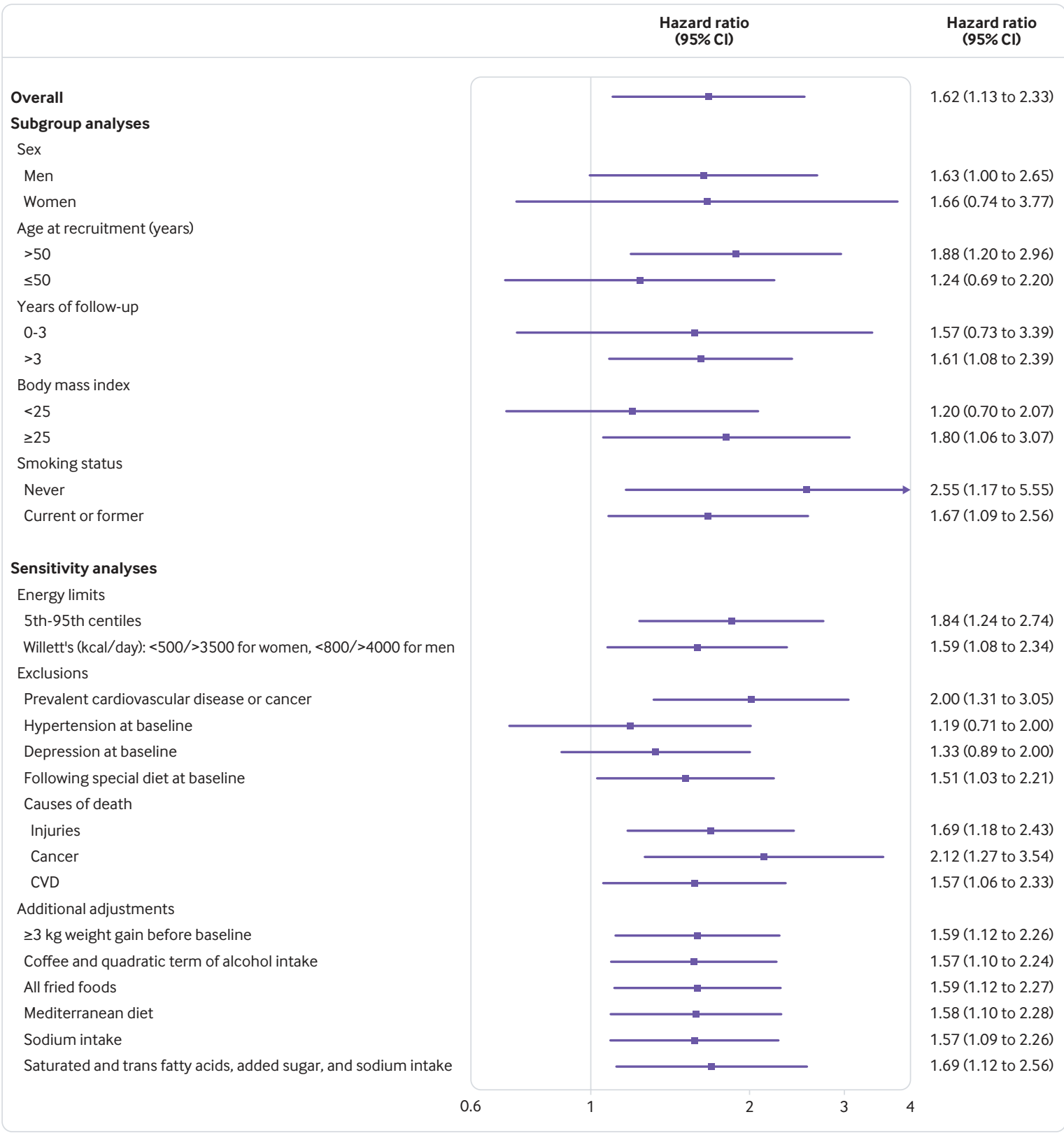

Fig 3 | Subgroup and sensitivity analyses for association between consumption of ultra-processed foods and all cause mortality (highest versus lowest quarter of consumption). See table 3 footnote $t$ for adjustment factors. $C V D=$ cardiovascular disease

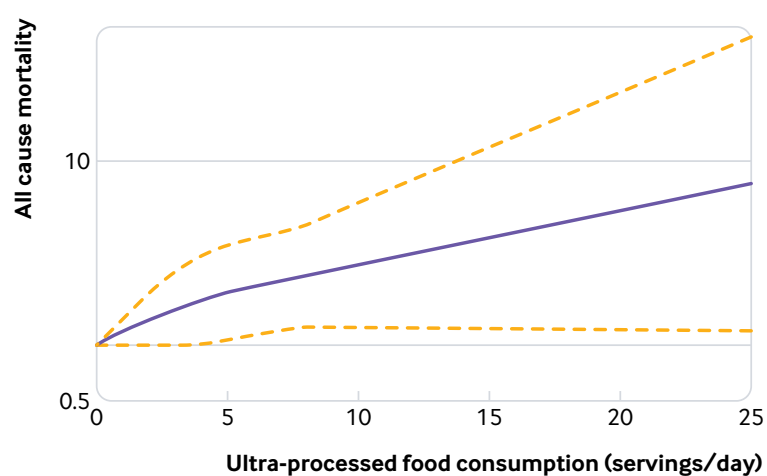

Fig 4 | Restricted cubic splines analysis of association between consumption of ultra-processed foods and all cause mortality. Dotted lines represent $95 \%$ confidence intervals potential confounding by socioeconomic status, but we are confident that this was not a major confounder in our analyses because our cohort was homogeneous for this variable. Adjustment for educational level is recommended to adjust for socioeconomic status, but our participants had attained the same educational level (ie, we used restriction instead of multivariate adjustment to control for confounding by socioeconomic status).

\section{Conclusions and policy implications}

Our results suggest that an increased consumption of ultra-processed food is associated with a higher hazard of all cause mortality. Improving diet based on adherence to minimally processed food-a key aspect of the Mediterranean diet-has been shown 
to protect against chronic disease ${ }^{45}$ and all cause mortality. ${ }^{46-49}$ Discouraging the consumption of ultraprocessed foods; targeting products, taxation, and marketing restrictions on ultra-processed products; and promotion of fresh or minimally processed foods, should be considered part of important health policy to improve global public health.

We thank the participants of the SUN cohort who answered our questionnaires biannually and Maria Soledad Hershey for help with proofreading the manuscript.

Contributors: AR-C, MAM-G, and MB-R conceived and designed the study. AR-C, IA-A, MAM-G, and MB-R analysed the data. CF-A and RDM acquired and analysed the data. MAM-G and MB-R obtained funding. $A R-C$ and CG-D drafted the manuscript. AR-C, MAM-G, IA-A, RDM, CF-A, $C G-D$, and $M B-R$ critically revised the manuscript. All authors gave final approval and agree to be accountable for all aspects of the work ensuring integrity and accuracy. The corresponding author attests that all listed authors meet authorship criteria and that no others meeting the criteria have been omitted. MB- $R$ is the guarantor.

Funding: The SUN Project is supported by the Spanish GovernmentInstituto de Salud Carlos III, and the European Regional Development Fund (FEDER) (RD 06/0045, PI14/01798, PI14/01764, PI17/01795), Navarra Regional Government, and the University of Navarra. The funding sources had no role in the design, execution, analyses, and interpretation of the data or decision to submit the results of this study.

Competing interests: All authors have completed the ICMJE uniform disclosure form at www.icmje.org/coi_disclosure.pdf and declare: no support from any organisation for the submitted work; no financial relationships with any organisations that might have an interest in the submitted work in the previous three years; no other relationships or activities that could appear to have influenced the submitted work.

Ethical approval: The protocol was written in accordance with the principles of the Declaration of Helsinki and was approved by the University of Navarra institutional review board.

Data sharing: No additional data available.

Transparency: The manuscript's guarantor (MB-R) affirms that this manuscript is an honest, accurate, and transparent account of the study being reported; that no important aspects of the study have been omitted; and that any discrepancies from the study as planned (and, if relevant, registered) have been explained.

This is an Open Access article distributed in accordance with the Creative Commons Attribution Non Commercial (CC BY-NC 4.0) license, which permits others to distribute, remix, adapt, build upon this work non-commercially, and license their derivative works on different terms, provided the original work is properly cited and the use is non-commercial. See: http://creativecommons.org/licenses/ by-nc/4.0/.

1 Mozaffarian D. Dietary and Policy Priorities for Cardiovascular Disease, Diabetes, and Obesity: A Comprehensive Review. Circulation 2016;133:187-225. doi:10.1161/ CIRCULATIONAHA.115.018585

2 Gakidou E, Afshin A, Abajobir AA, et al, GBD 2016 Risk Factors Collaborators. Global, regional, and national comparative risk assessment of 84 behavioural, environmental and occupational, and metabolic risks or clusters of risks, 1990-2016: a systematic analysis for the Global Burden of Disease Study 2016. Lancet 2017:390:1345-422. doi:10.1016/S01406736(17)32366-8

3 United Nations. Political declaration of the high-level meeting of the General Assembly on the prevention and control of noncommunicable diseases. Agenda item 117. 2011; (January). doi:10.1007/BF03038934

4 World Health Organization. Global action plan for the prevention and control of noncommunicable diseases 2013-2020. Geneva: WHO www.who.int/nmh/events/ncd_action_plan/en/. Published 2013.

5 Kontis V, Mathers CD, Rehm J, et al. Contribution of six risk factors to achieving the $25 \times 25$ non-communicable disease mortality reduction target: a modelling study. Lancet 2014;384:427-37. doi:10.1016/ S0140-6736(14)60616-4

6 Moodie R, Stuckler D, Monteiro C, et al, Lancet NCD Action Group. Profits and pandemics: prevention of harmful effects of tobacco, alcohol, and ultra-processed food and drink industries. Lancet 2013;381:670-9. doi:10.1016/S0140-6736(12)62089-3
7 Monteiro CA, Moubarac JC, Cannon G, Ng SW, Popkin B. Ultraprocessed products are becoming dominant in the global food system. Obes Rev 2013;14(Suppl 2):21-8. doi:10.1111/ obr.1210710.1111/obr.12107

8 Latasa P, Louzada MLDC, Martinez Steele E, Monteiro CA. Added sugars and ultra-processed foods in Spanish households (19902010). Eur J Clin Nutr 2018;72:1404-12. doi:10.1038/s41430017-0039-0

9 Monteiro CA, Cannon G, Levy R, et al. NOVA. The star shines bright. Food classification. Public Health World Nutr 2016;7:28-38

10 Moubarac JC, Martins APB, Claro RM, Levy RB, Cannon G, Monteiro CA, Evidence from Canada. Consumption of ultraprocessed foods and likely impact on human health. Public Health Nutr 2013;16:2240-8. doi:10.1017/S1368980012005009

11 Singh GM, Micha R, Khatibzadeh S, Lim S, Ezzati M, Mozaffarian D, Global Burden of Diseases Nutrition and Chronic Diseases Expert Group (NutriCoDE). Estimated Global, Regional, and National Disease Burdens Related to Sugar-Sweetened Beverage Consumption in 2010. Circulation 2015;132:639-66. doi:10.1161/ CIRCULATIONAHA.114.010636

12 Larsson SC, Orsini N. Red meat and processed meat consumption and all-cause mortality: a meta-analysis. Am J Epidemiol 2014:179:282-9. doi:10.1093/aje/kwt261

13 Wang X, Lin X, Ouyang YY, et al. Red and processed meat consumption and mortality: dose-response meta-analysis of prospective cohort studies. Public Health Nutr 2016;19:893-905. doi:10.1017/S1368980015002062

14 Fiolet T, Srour B, Sellem L, et al. Consumption of ultra-processed foods and cancer risk: results from NutriNet-Santé prospective cohort. BMJ 2018;360:k322. doi:10.1136/bmi.k322

15 Schnabel L, Buscail C, Sabate JM, et al. Association Between Ultra-Processed Food Consumption and Functional Gastrointestinal Disorders: Results From the French NutriNet-Santé Cohort. Am J Gastroenterol 2018;113:1217-28. doi:10.1038/s41395-018 0137-1

16 Rauber F, Campagnolo PDB, Hoffman DJ, Vitolo MR. Consumption of ultra-processed food products and its effects on children's lipid profiles: a longitudinal study. Nutr Metab Cardiovasc Dis 2015;25:116-22. doi:10.1016/j.numecd.2014.08.001

17 Mendonça RD, Pimenta AM, Gea A, et al. Ultraprocessed food consumption and risk of overweight and obesity: the University of Navarra Follow-Up (SUN) cohort study. Am J Clin Nutr 2016:104:1433-40. doi:10.3945/ajcn.116.135004

18 Mendonça RD, Lopes AC, Pimenta AM, Gea A, Martinez-Gonzalez MA, Bes-Rastrollo M. Ultra-processed food consumption and the incidence of hypertension in a mediterranean cohort: The seguimiento universidad de navarra project. $A m$ J Hypertens 2017;30:358-66.

19 Schnabel L, Kesse-Guyot E, Allès B, et al. Association Between Ultraprocessed Food Consumption and Risk of Mortality Among Middle-aged Adults in France. JAMA Intern Med 2019. doi:10.1001/ jamainternmed.2018.7289

$20 \mathrm{Kim} \mathrm{H}, \mathrm{Hu}$ EA, Rebholz CM. Ultra-processed food intake and mortality in the USA: results from the Third National Health and Nutrition Examination Survey (NHANES III, 1988-1994). Public Health Nutr 2019:1-9.

21 Carlos S, De La Fuente-Arrillaga C, Bes-Rastrollo M, et al. Mediterranean diet and health outcomes in the SUN cohort. Nutrients 2018;10:E439. doi:10.3390/nu10040439

22 Martín-Moreno JM, Boyle P, Gorgojo L, et al. Development and validation of a food frequency questionnaire in Spain. Int J Epidemiol 1993;22:512-9. doi:10.1093/ije/22.3.512

23 de la Fuente-Arrillaga C, Ruiz ZV, Bes-Rastrollo M, Sampson L, Martinez-González MA. Reproducibility of an FFQ validated in Spain. Public Health Nutr 2010;13:1364-72. doi:10.1017/ S1368980009993065

24 Fernández-Ballart JD, Piñol JL, Zazpe I, et al. Relative validity of a semi-quantitative food-frequency questionnaire in an elderly Mediterranean population of Spain. Br J Nutr 2010;103:1808-16. doi:10.1017/S0007114509993837

25 Trichopoulou A, Costacou T, Bamia C, Trichopoulos D. Adherence to a Mediterranean diet and survival in a Greek population. N Engl I Med 2003;348:2599-608. doi:10.1056/NEJMoa02503910.1056/ NEJMoa025039

26 Martínez-González MA, López-Fontana C, Varo JJ, Sánchez-Villegas A, Martinez JA. Validation of the Spanish version of the physical activity questionnaire used in the Nurses' Health Study and the Health Professionals' Follow-up Study. Public Health Nutr 2005;8:920-7. doi:10.1079/PHN200574510.1079/PHN2005745

27 Bes-Rastrollo M, Pérez-Valdivieso JR, Sánchez-Villegas A, et al. Validación del peso e índice de masa corporal auto-declarados de los participantes de una cohorte de graduados universitarios. Rev Esp Obes 2005:3:352-8.

28 Hernán MARJ. Causal Inference. Chapman \& Hall/CRC, 2019, (forthcoming) 
29 Hernán MA, Hernández-Díaz S, Werler MM, Mitchell AA Causal knowledge as a prerequisite for confounding evaluation: an application to birth defects epidemiology. Am Epidemiol 2002;155:176-84. doi:10.1093/aje/155.2.176

30 Greenland S, Daniel R, Pearce N. Outcome modelling strategies in epidemiology: traditional methods and basic alternatives. Int I Epidemiol 2016;45:565-75. doi:10.1093/ije/dyw040

31 VanderWeele TJ, Ding P. Sensitivity Analysis in Observational Research: Introducing the E-Value. Ann Intern Med 2017;167:26874. doi:10.7326/M16-2607

32 Mathur MB, Ding P, Riddell CA, VanderWeele TJ. Web Site and R Package for Computing E-values. Epidemiology 2018;29:e45-7. doi:10.1097/EDE.0000000000000864

33 Willett W. Nutritional Epidemiology. 3rd ed. Oxford University Press, 2013.

34 Sarkar SK, Chang CK, Chang CK. The simes method for multiple hypothesis testing with positively dependent test statistics. J Am Sta Assoc 1997;92:1601- 8. doi:10.1080/01621459.1997.10473682

35 Gibney MJ, Forde CG, Mullally D, Gibney ER. Ultra-processed foods in human health: a critical appraisal. Am J Clin Nutr 2017;106:717-24.

36 Adams J, White M. Characterisation of UK diets according to degree of food processing and associations with socio-demographics and obesity: cross-sectional analysis of UK National Diet and Nutrition Survey (2008-12). Int J Behav Nutr Phys Act 2015;12:160. doi:10.1186/s12966-015-0317-y

37 Martínez Steele E, Monteiro CA. Association between dietary share of ultra-processed foods and urinary concentrations of phytoestrogens in the US. Nutrients 2017;9:209. doi:10.3390/nu9030209

38 Rohatgi KW, Tinius RA, Cade WT, Steele EM, Cahill AG, Parra DC. Relationships between consumption of ultra-processed foods, gestational weight gain and neonatal outcomes in a sample of US pregnant women. Peer) 2017;5:e4091. doi:10.7717/peerj.4091

39 Poti JM, Mendez MA, Ng SW, Popkin BM. Is the degree of food processing and convenience linked with the nutritional quality of foods purchased by US households?Am J Clin Nutr 2015;101:125162. doi:10.3945/ajen.114.100925
40 Martínez Steele E, Baraldi LG, Louzada ML, Moubarac JC, Mozaffarian D, Monteiro CA. Ultra-processed foods and added sugars in the US diet: evidence from a nationally representative cross-sectional study. BMJ Open 2016;6:e009892. doi:10.1136/bmjopen-2015-009892

41 Bielemann RM, Santos LP, Costa CDS, Matijasevich A, Santos IS. Early feeding practices and consumption of ultraprocessed foods at $6 y$ of age: Findings from the 2004 Pelotas (Brazil) Birth Cohort Study. Nutrition 2018;47:27-32. doi:10.1016/j.nut.2017.09.012

42 Rothman KJ, Gallacher JEJ, Hatch EE. Why representativeness should be avoided. Int J Epidemiol 2013;42:1012-4. doi:10.1093/ije/ dys 223

43 Rothman K, Greenland S, Lash T. Modern Epidemiology. 3rd edn. Lippincott Williams \& Wilkins. Philadelphia.

44 Willett WC, Colditz GA. Approaches for conducting large cohort studies. Epidemiol Rev 1998;20:91-9. doi:10.1093/oxfordjournals. epirev.a017975

45 Martínez-González MA, Gea A, Ruiz-Canela M. The Mediterranean Diet and Cardiovascular Health. Circ Res 2019;124:77998. doi:10.1056/NEJMoa1800389. doi:10.1161/ CIRCRESAHA.118.313348

46 Martínez-González MA, Guillén-Grima F, De Irala J, et al. The Mediterranean diet is associated with a reduction in premature mortality among middle-aged adults. J Nutr 2012;142:1672-8. doi:10.3945/jn.112.162891

47 Chen G-C, Zhang R, Martínez-González MA, et al. Nut consumption in relation to all-cause and cause-specific mortality: a meta-analysis 18 prospective studies. Food Funct 2017;8:3893-905. doi:10.1039/ C7FO00915A

48 Reedy J, Krebs-Smith SM, Miller PE, et al. Higher diet quality is associated with decreased risk of all-cause, cardiovascular disease, and cancer mortality among older adults. J Nutr 2014;144:881-9. doi:10.3945/jn.113.189407

49 Alvarez-Alvarez I, Zazpe I, Pérez de Rojas J, et al. Mediterranean diet, physical activity and their combined effect on all-cause mortality: The Seguimiento Universidad de Navarra (SUN) cohort. Prev Med 2018;106:45-52. doi:10.1016/i.ypmed.2017.09.02 